\title{
Supervivencia de los pacientes receptores de trasplante renal
}

\author{
Marco Antonio Ayala-García, ${ }^{*}$ Ernesto Díaz-Chávez, ${ }^{2}$ Joel Máximo Soel-Encalada, ${ }^{2}$ \\ Abel Orozco-Mosqueda, ${ }^{2}$ María Haydée Balandrán-Ortiz, ${ }^{3}$ Silvia Rebeca Ceja-Guzmán, ${ }^{4}$ \\ Saulit Garcidueñas-Garcidueñas, ${ }^{5}$ Gilberto Flores-Vargas ${ }^{6}$ y Eduardo Guaní-Guerra ${ }^{7}$ \\ ${ }^{1}$ Instituto Mexicano del Seguro Social, Hospital General Regional 58, Departamento de Cirugía; ${ }^{2}$ Secretaría de Salud, Hospital Regional de Alta \\ Especialidad del Bajío, Unidad de Trasplantes; ${ }^{3}$ Secretaría de Salud del Estado de Guanajuato, Hospital General de Irapuato, Servicio de Consulta \\ Externa; ${ }^{4}$ Secretaría de Salud del Estado de Guanajuato, Hospital General de Pénjamo, Departamento de Calidad; ${ }^{5}$ Secretaría de Salud del Estado \\ de Guanajuato, Centro de Salud con Servicios Extendidos, Departamento de Epidemiología; ${ }^{6}$ Centro de Investigaciones en Matemáticas A. C.; \\ ${ }^{7}$ Secretaría de Salud, Hospital Regional de Alta Especialidad del Bajío, Unidad de Investigación. Guanajuato, México
}

\section{Resumen}

Introducción: El Hospital Regional de Alta Especialidad del Bajío inició sus funciones en 2007 para atender la demanda de salud de 5.8 millones de habitantes, cuenta con 184 camas y una unidad de trasplantes con 26 camas. En 2008 inició actividades el programa de trasplante renal. Objetivo: Presentar la supervivencia de los pacientes receptores de trasplante renal y del riñón injertado en el Hospital Regional de Alta Especialidad del Bajío, Guanajuato, México. Método: Estudio de cohorte retrospectivo en el que se incluyeron los trasplantes consecutivos realizados entre 2008 y 2016 . El análisis estadístico se efectuó con el método de Kaplan-Meier. Resultados: Se analizaron 837 trasplantes. La supervivencia del injerto censurada para muerte con injerto funcional a uno y cinco años fue de 94.6 y $78.9 \%$. La supervivencia del paciente a uno y cinco años fue de 95.4 y $88.1 \%$. Conclusiones: El programa de trasplante renal constituye uno de los mejor establecidos en México, tanto por el número de trasplantes renales de donante fallecido realizados como por la supervivencia obtenida de paciente e injerto. Los datos indican que el programa de trasplante renal ha tenido un desarrollo sostenido.

PALABRAS CLAVE: Trasplante Renal. Supervivencia paciente. Supervivencia injerto.

\section{Renal transplantation recipient patients survival}

\section{Abstract}

Introduction: The Bajío High Specialty Regional Hospital started operating in 2007 to tackle the health demands of 5.8 million inhabitants. It has 184 beds and a transplant unit with 26 beds. In 2008, the renal transplant program launched activities. Objective: To describe the survival of kidney transplant receptor patients and of the grafted kidney at the Bajío High Specialty Regional Hospital. Methods: Retrospective cohort study, where consecutive transplants carried out between 2008 and 2016 were included. Statistical analysis was performed using the Kaplan-Meier method. Results: $A$ total of 837 transplants were analyzed. Graft survival censored for death, with a functional graft at 1 and 5 years, was 94.6\% and 78.9\%. Patient survival at 1 and 5 years was $95.4 \%$ and $88.1 \%$. Conclusions: The renal transplant program is one of the the best programs established in Mexico, both for the number of deceased-donor kidney transplants performed and for the patient and graft survival achieved. These data indicate that the renal transplant program has had a sustained development.

KEY WORDS: Renal transplant. Patient survival. Graft survival.

Correspondencia:

*Marco Antonio Ayala-García

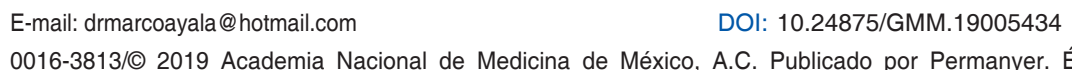

CC BY-NC-ND (http://creativecommons.org/licenses/by-nc-nd/4.0/).
Fecha de recepción: 29-07-2019

Fecha de aceptación: 07-09-2019

DOI: 10.24875/GMM.19005434
Gac Med Mex. 2020;156:34-39

Disponible en PubMed

www.gacetamedicademexico.com 


\section{Introducción}

El trasplante renal es la mejor alternativa de tratamiento para la insuficiencia renal crónica terminal. ${ }^{1}$ En 1954 se realizó el primer trasplante renal exitoso en humanos (Boston, Estados Unidos). En México, el primer trasplante renal se efectuó el 22 de octubre de 1963 en el Hospital General del Centro Médico Nacional del Instituto Mexicano del Seguro Social. ${ }^{2}$ Actualmente, en México hay 248 centros hospitalarios autorizados para llevar a cabo trasplante renal, sin embargo, solo 142 tienen actividad; 3 en 2017, el Hospital Regional de Alta Especialidad del Bajío (HRAEB) ocupó el cuarto lugar nacional entre los establecimientos con el mayor número de trasplantes con 129 trasplantes durante el año y el primer lugar en trasplantes de donador fallecido. ${ }^{4}$ La tasa nacional de donación en 2016 fue de 32.2 por millón de habitantes; la de donador fallecido, de 15.5 por millón de habitantes; y la de donador con muerte encefálica, de 4.1 por millón de habitantes. ${ }^{5}$ En los últimos cinco años, anualmente en México se realiza un promedio de 2901 trasplantes renales y existen 12477 pacientes en espera de un riñón. ${ }^{3}$

EI HRAEB es público y recibe recursos federales, inició sus funciones en 2007 para atender la demanda de salud de aproximadamente 5.8 millones de habitantes de los estados de Guanajuato, Jalisco, Michoacán, Aguascalientes y Zacatecas. Es un hospital de tercer nivel localizado en León, Guanajuato, en el Centro Occidente de México, que dispone de 184 camas, incluyendo 16 en terapia intensiva, 15 en urgencias y 26 en la unidad de trasplantes; en 2008 inició el programa de trasplante renal y a partir de 2014 cuenta con la residencia médica para formar especialistas en cirugía de trasplante renal. ${ }^{6}$

El presente documento expone la supervivencia de los pacientes receptores de trasplante renal y la del riñón injertado, y la información epidemiológica relevante relativa a los pacientes receptores de un trasplante renal en el HRAEB.

\section{Método}

Estudio de cohorte retrospectivo de 837 trasplantes renales realizados del 26 de enero de 2008 al 31 de diciembre de 2016. Se analizaron las siguientes variables: edad, sexo, tipo de donador, etiología de la enfermedad renal, inmunosupresión, derechohabiencia, entidad federativa de origen, mortalidad y pérdida del injerto. Se obtuvieron medidas de tendencia central y dispersión (media y desviación estándar) para las variables cuantitativas y proporciones para las cualitativas. El análisis de supervivencia se realizó con el método de Kaplan-Meier. Se muestra la supervivencia del paciente y la del injerto censurada para muerte con injerto funcional. Para establecer las diferencias en la supervivencia de acuerdo con el tipo de donador se empleó la prueba de log-rank; el nivel de significación se estableció en $p<0.05$. Toda la información procedió de la base de datos de la Unidad de Trasplantes del HRAEB, del expediente electrónico de cada paciente y del archivo físico.

\section{Resultados}

Se analizaron los datos de los primeros nueve años del programa de trasplante renal en el HRAEB, es decir, 837 trasplantes renales realizados entre el 26 de enero de 2008 y el 31 de diciembre de 2016. De los pacientes receptores de trasplante renal solo cuatro recibieron un segundo injerto renal. Se efectuaron $409(48.8 \%)$ trasplantes de donante fallecido $\mathrm{y}$ $428(51.1 \%)$ de donador vivo. La edad media \pm desviación estándar de los pacientes receptores de trasplante renal fue de $26.2 \pm 12.1$ años, con un rango de 3 a 69.

En cuanto a los donadores fallecidos, la edad media fue de $28.6 \pm 15$ años, con un rango de 0 a 65 . En relación con los donadores vivos, 385 fueron relacionados y 43 no relacionados. La edad media para los donadores vivos fue de $36.4 \pm 10.3$ años, con un rango de 18 a 54. Al comparar las edades de los donadores se encontró una diferencia significativa $(p<0.001)$ entre los fallecidos y los vivos.

La etiología de la insuficiencia renal fue desconocida en 631 de los receptores (75.3\%). Se utilizó inducción para la inmunosupresión en 589 pacientes (70.4\%). El esquema de inmunosupresión más usado fue el de la ciclosporina. De los pacientes receptores de trasplante renal, $511(61 \%)$ no tenían seguridad social (Tabla 1) y $94.2 \%$ fue originario del estado de Guanajuato; el municipio de origen se indica en la Figura 1.

Se registró una mortalidad de 8.2 \% (69 defunciones de 837 trasplantados); las causas de muerte se describen en la Tabla 2. Se perdieron 111 injertos (13.2 \% de los trasplantados).

La supervivencia del injerto censurada para muerte con injerto funcional a uno, tres, cinco y ocho años fue de $94.6,88.6,78.9$ y $69.4 \%$. En la Figura 2 se 
Tabla 1. Características de 837 pacientes en quienes se efectuó trasplante del Hospital Regional de Alta Especialidad del Bajío

\begin{tabular}{|c|c|c|}
\hline Variable & \multicolumn{2}{|c|}{ Media \pm DE } \\
\hline Edad de receptor (años) & \multicolumn{2}{|c|}{$26.2 \pm 12.1$} \\
\hline & $n$ & $\%$ \\
\hline Receptor masculino & 575 & 68.6 \\
\hline Donador fallecido & 409 & 48.8 \\
\hline Donador vivo relacionado & 385 & 45.9 \\
\hline Donador vivo no relacionado & 43 & 5.1 \\
\hline $\begin{array}{l}\text { Etiología de la enfermedad renal } \\
\text { Desconocida } \\
\text { Glomerulopatía secundaria } \\
\text { Glomerulopatía primaria } \\
\text { Nefropatía por malformación del tracto urinario } \\
\text { Enfermedad poliquística } \\
\text { Glomerulopatía congénita }\end{array}$ & $\begin{array}{l}631 \\
106 \\
57 \\
20 \\
20 \\
3\end{array}$ & $\begin{array}{l}75.3 \\
12.6 \\
6.8 \\
2.3 \\
2.3 \\
0.3\end{array}$ \\
\hline $\begin{array}{l}\text { Inmunosupresión } \\
\text { Inducción con basiliximab } \\
\text { Inducción con timoglobulina } \\
\text { Inducción con daclizumab } \\
\text { Sin inducción } \\
\text { Ciclosporina-micofenolato de mofetilo-prednisona } \\
\text { Tacrolimus-micofenolato de mofetilo-prednisona } \\
\text { Sin inmunosupresión por defunción }\end{array}$ & $\begin{array}{c}492 \\
92 \\
5 \\
248 \\
542 \\
290 \\
5\end{array}$ & $\begin{array}{r}58.7 \\
10.9 \\
0.5 \\
29.6 \\
64.7 \\
34.6 \\
0.5\end{array}$ \\
\hline $\begin{array}{l}\text { Derechohabiencia } \\
\text { Sin seguridad social } \\
\text { Instituto Mexicano del Seguro Social } \\
\text { Instituto de Seguridad y Servicios Sociales de los Trabajadores del Estado } \\
\text { Secretaría de la Defensa Nacional }\end{array}$ & $\begin{array}{c}511 \\
315 \\
10 \\
1\end{array}$ & $\begin{array}{c}61 \\
37.6 \\
1.1 \\
0.1\end{array}$ \\
\hline $\begin{array}{l}\text { Entidad federativa de origen } \\
\text { Guanajuato } \\
\text { Jalisco } \\
\text { Zacatecas } \\
\text { Michoacán } \\
\text { San Luis Potosí } \\
\text { Querétaro } \\
\text { Distrito Federal }\end{array}$ & $\begin{array}{c}789 \\
21 \\
15 \\
7 \\
2 \\
2 \\
1\end{array}$ & $\begin{array}{l}94.2 \\
2.5 \\
1.7 \\
0.8 \\
0.2 \\
0.2 \\
0.1\end{array}$ \\
\hline
\end{tabular}

muestran las curvas de Kaplan-Meier de la supervivencia del injerto censurada para muerte con injerto funcional de acuerdo con el tipo de donador y la prueba de log-rank fue usada para comparar ambos grupos de acuerdo con el tipo de donador para la supervivencia del injerto a nueve años, encontrando una diferencia estadísticamente significativa $(p=0.002)$.

La supervivencia censurada del paciente receptor de trasplante renal a uno, tres, cinco y ocho años fue de $95.4,92,88.1$ y $84.4 \%$, respectivamente. En la Figura 3 se presentan las curvas de Kaplan-Meier de la supervivencia del paciente receptor de trasplante renal de acuerdo con el tipo de donador y la prueba de log-rank fue usada para comparar ambos grupos de acuerdo con el tipo de donador para la supervivencia del receptor a nueve años, con lo que se encontró una diferencia estadísticamente significativa $(p=0.003)$.

\section{Discusión}

Se presentan los resultados del programa de trasplante renal en el HRAEB durante sus primeros nueve años. En 2016, en el HRAEB se llevó a cabo $3.2 \%$ de los trasplantes renales realizados en México (98 de 2970 trasplantes renales) y $6.7 \%$ de los trasplantes renales de donador fallecido (57 trasplantes en el HRAEB, de 844 realizados en el país), con lo que ocupó el primer lugar en México de los establecimientos con mayor actividad de trasplante renal de donante fallecido. ${ }^{5}$ 


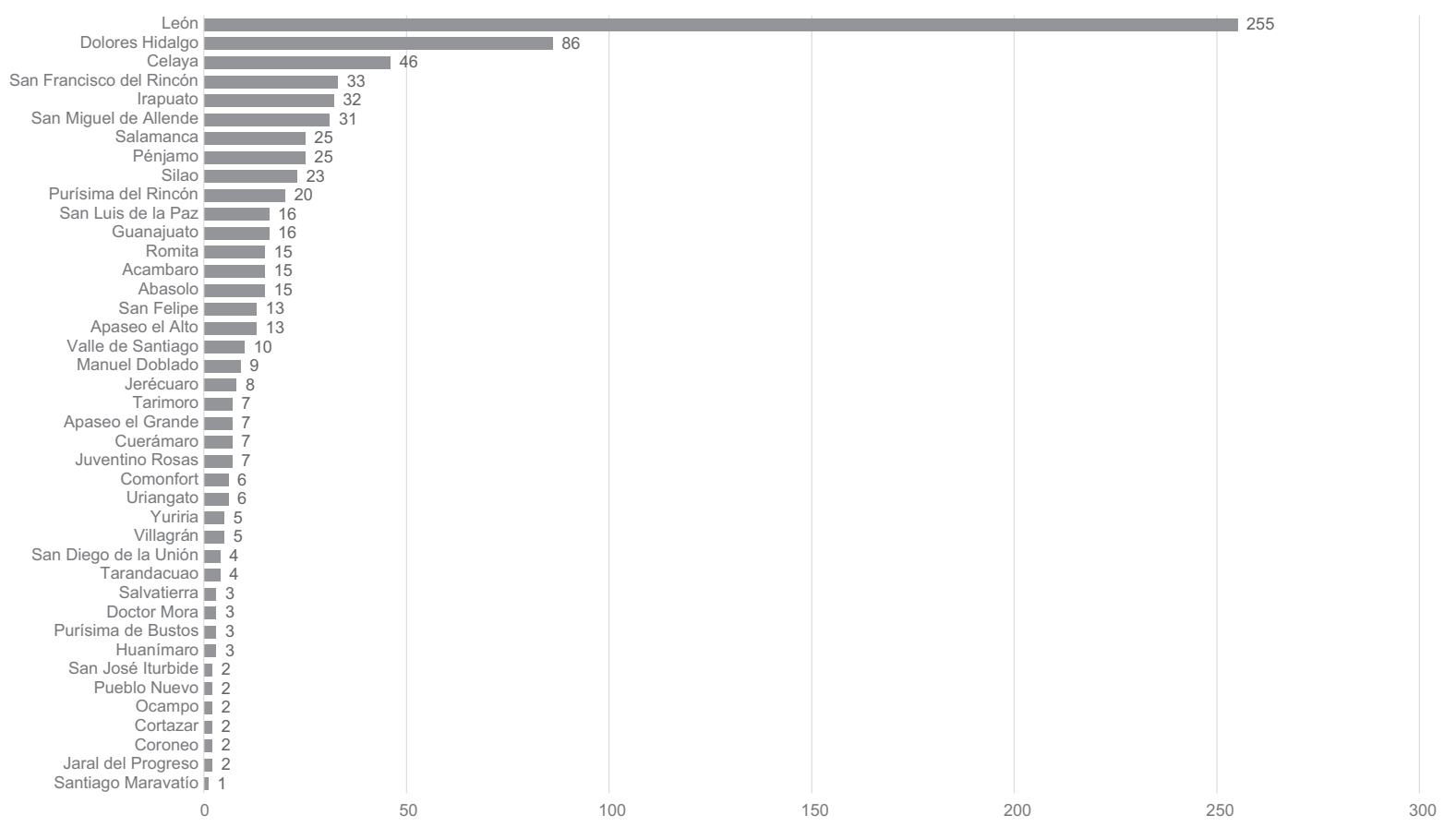

Figura 1. Municipio de origen en el estado de Guanajuato de los pacientes con trasplante renal en el Hospital Regional de Alta Especialidad del Bajío $(n=789)$.

Tabla 2. Causas de muerte de 69 pacientes con trasplante renal

\begin{tabular}{|l|c|c|}
\hline Causa & $n$ & $\%$ \\
\hline Insuficiencia renal terminal (falla del injerto) & 26 & 37.6 \\
\hline Rechazo agudo del injerto & 14 & 20.2 \\
\hline Nefropatía crónica del injerto & 6 & 8.6 \\
\hline Desconocida & 3 & 4.3 \\
\hline Infección y posterior remoción del injerto & 2 & 2.9 \\
\hline Disfunción primaria del injerto & 1 & 1.4 \\
\hline Infecciosas & 26 & 37.6 \\
\hline Neoplasias & 7 & 10.1 \\
\hline Enfermedad pulmonar no infecciosa & 2 & 2.8 \\
\hline Infarto agudo al miocardio & 2 & 2.8 \\
\hline Hemorragia & 2 & 2.8 \\
\hline Suicidio & 2 & 2.8 \\
\hline Complicaciones de la diabetes mellitus & 1 & 1.4 \\
\hline Homicidio & 1 & 1.4 \\
\hline
\end{tabular}

El número de trasplantes renales realizados en hospitales similares en México, como el Instituto Nacional de Ciencias Médicas y Nutrición "Salvador Zubirán", el cual en 44 años (1967-2011) hizo 1000 trasplantes renales $^{7}$ y el Hospital Regional de Alta Especialidad de Veracruz, el cual en un periodo de 10 años (2006-2016) efectuó 95 trasplantes renales, ${ }^{8}$ contrasta con los 837 realizados en nueve años (2008-2016) en el HRAEB, que lo convierten en uno de los hospitales con mayor importancia en México respecto a este procedimiento.

EI HRAEB es un hospital regional, es decir, debe cubrir las demandas de los habitantes de Guanajuato, Jalisco, Michoacán, Aguascalientes y Zacatecas. Sin embargo, $94.2 \%$ de los pacientes receptores de trasplante renal son de Guanajuato y $30.4 \%$, es decir, 255 de los receptores del estado de Guanajuato, pertenecen al municipio de León, lo que se explica por la ubicación de este nosocomio en León.

En el programa de trasplantes del HRAEB han sido atendidos pacientes de 41 de los 46 municipios del estado de Guanajuato, pero no hay pacientes de Atarjea, Tierra Blanca, Xichú, Santa Catarina y Victoria, municipios ubicados en el noreste del estado de Guanajuato, lejos del HRAEB, lo que puede ser una de las razones para que los pacientes de esos municipios no sean referidos, no acudan al HRAEB y sean atendidos en otros hospitales como el Hospital Central "Dr. Ignacio Morones Prieto", en San Luis Potosí (si son derechohabientes del Instituto Mexicano del Seguro Social) o el Hospital Regional 1 de Querétaro.

Los pacientes receptores de trasplante renal en el HRAEB fueron adultos jóvenes con edad promedio de 26.2 años, similar a la reportada en el Instituto 


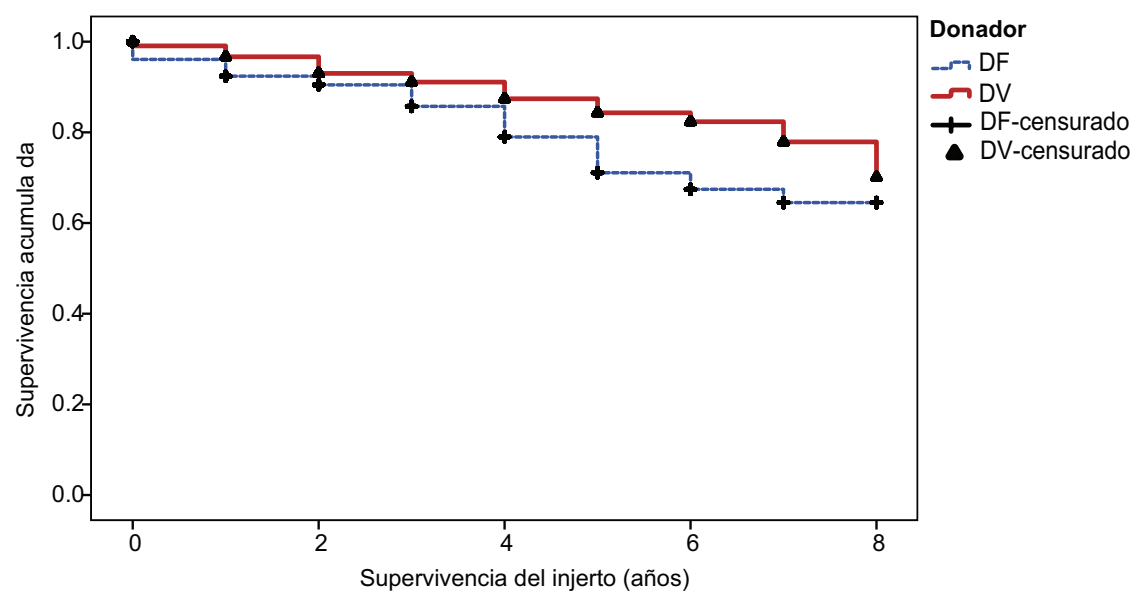

Figura 2. Curvas de Kaplan-Meier para la supervivencia del injerto renal trasplantado en el del Hospital Regional de Alta Especialidad del Bajío. $D V=$ donador vivo, $D F=$ donador fallecido .

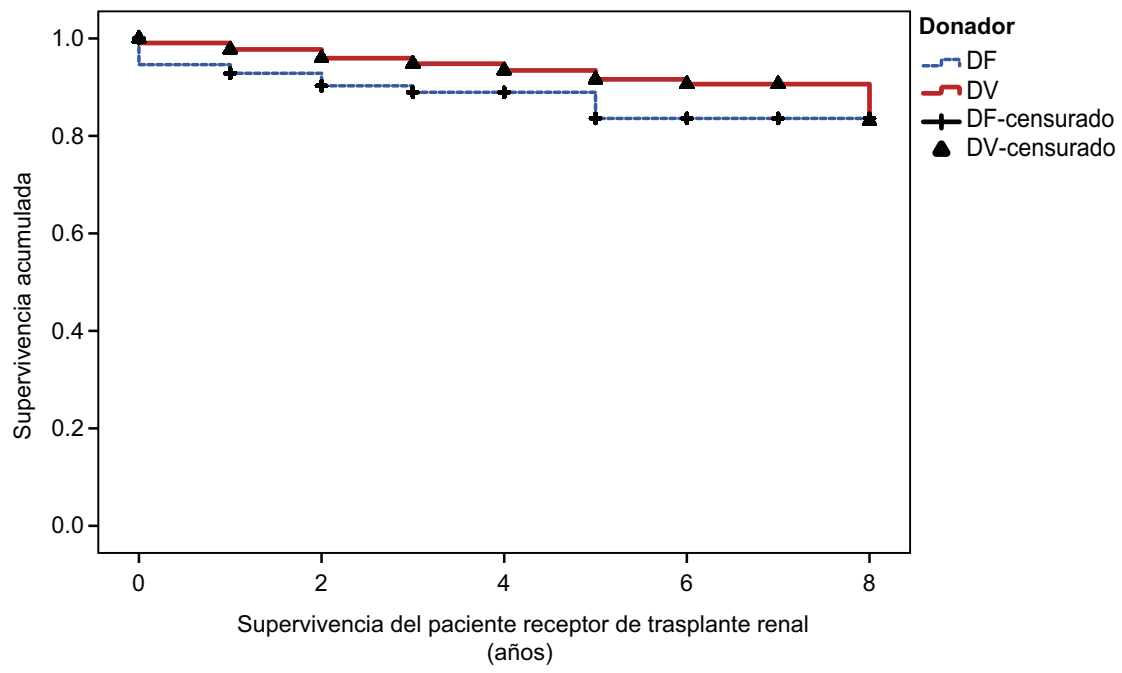

Figura 3. Curvas de Kaplan-Meier para la supervivencia del paciente receptor de trasplante renal en el del Hospital Regional de Alta Especialidad del Bajío. $D V=$ donador vivo, $D F=$ donador fallecido.

Nacional de Ciencias Médicas y Nutrición "Salvador Zubirán" (31.7 \pm 11.3 años), ${ }^{7}$ Hospital Regional de Alta Especialidad de Veracruz (31.4 \pm 12.8 años), ${ }^{8}$ Instituto Nacional de Cardiología "Ignacio Chávez"(29.9 \pm 11.6 años), ${ }^{9}$ Hospital de Especialidades "Bernardo Sepúlveda" del Centro Médico Nacional Siglo XXI (31.4 \pm 10.5 años), ${ }^{1}$ Hospital Miguel Hidalgo en Aguascalientes $\left(29.3 \pm 15\right.$ años) ${ }^{10}$ Hospital Central "Dr. Ignacio Morones Prieto" (26.8 \pm 16.6 años $)^{11} \mathrm{e}$ Instituto Mexicano de Trasplantes (31 \pm 10 años)..$^{12}$

La etiología de la insuficiencia renal terminal se desconoció en $75.3 \%$ de los casos, debido a que se trata de un hospital de referencia y la mayoría de los pacientes atendidos se encontraba en etapas tardías de la enfermedad, por lo que la biopsia renal con fines diagnósticos ya no fue una opción. Para evitar lo anterior es necesario implementar programas de detección temprana, en hospitales y centros de salud de primer contacto.

Durante el periodo reportado, en el HRAEB solo se usaron dos esquemas de inmunosupresión: ciclosporina-micofenolato de mofetilo-prednisona y tacrolimus-micofenolato de mofetilo-prednisona.

Las comparaciones con los datos reportados en la literatura en relación con la supervivencia de los pacientes trasplantados renales y del injerto en México son limitadas por la variabilidad en la metodología de análisis y tratamiento de los datos, así como por analizar cohortes con diferentes periodos,,$^{1,7-12}$ sin embargo, podemos señalar que existen diferencias $(p>0.05)$ respecto a la supervivencias del paciente con trasplante renal señalada en España: 91, 81 y 57 \% para 
uno, dos y tres años respectivamente, ${ }^{13}$ en contraste con las encontradas en este estudio: 95.4, 93.2 y $92 \%$, para esos mismos periodos.

En este estudio, la supervivencia del receptor de trasplante renal de donador vivo, tanto del paciente como del injerto, fue mayor en comparación con la del receptor de trasplante renal de donador fallecido, como se señala en otras investigaciones. ${ }^{1,7-12}$ Según distintos análisis, existen diversos factores que podrían contribuir a explicar la mejor supervivencia y filtrado glomerular de los trasplantes de donador vivo, ${ }^{14-16}$ como la tipificación rutinaria del grupo sanguíneo y las pruebas de histocompatibilidad que se realizan en el HRAEB:

- Tipificación HLA del donante y receptor, a través de biología molecular con reacción en cadena de la polimerasa tipo SSP (secuence-specific primers) para clases I y II (A, B, DRB1 y DQB1).

- Aloanticuerpos anti-HLA a través del panel reactivo de anticuerpos con la metodología ELISA (fase sólida).

- Prueba cruzada linfocitaria por citotoxicidad mediada por complemento, con el empleo de linfocitos totales y ditiotreitol.

Respecto a los donadores vivos, no contamos con los datos de compatibilidad HLA de $5.1 \%$ (22/428) donadores-receptores, $64.5 \%$ (276/428) coincidía en un haplotipo donador-receptor y $9.1 \%$ (39/428) coincidía en dos haplotipos donador-receptor, de tal forma que la identidad HLA (coincidencia de uno y dos haplotipos) contribuyó a que $73.6 \%$ (297/428) de los receptores de trasplante renal de donador vivo tuviera mejor supervivencia que la del receptor de trasplante renal de donador fallecido.

\section{Conclusiones}

El programa de trasplante renal del HRAEB constituye uno de los programas más sólidos en México, tanto por el número de trasplantes renales de donante fallecido realizados como por la supervivencia obtenida de paciente e injerto. A pesar de ser un programa relativamente reciente (nueve años) en un hospital con apenas 10 años de creación, la supervivencia del receptor y del injerto es mejor a la de otros hospitales con mayor experiencia en el procedimiento. Los datos también indican que el programa de trasplante renal ha tenido un desarrollo sostenido.

\section{Bibliografía}

1. Gracida-Juárez C, Espinoza-Pérez R, Cancino-López JD, Ibarra-Villanueva A, Cedillo-López U, Villegas-Anzo F, et al. Kidney transplant experience at the Specialty Hospital Bernardo Sepúlveda National Medical Center Century XXI, Mexican Institute of Social Security. Rev Invest Clin. 2011;63:19-24.

2. Quijano M, Gómez-Mont F, Ortiz-Quezada, Ronces R. First experiences in human renal transplantation. Gac Med Mex. 1964;94:93-105.

3. Aburto S. Behavior of the donation and procurement of organs and tissues; receptors waiting for transplant; authorized units for procurement of transplants. Bol Estad Inform. 2016;1:15-42.

4. Centro Nacional de Trasplantes. Estadísticas [en línea]. México: Centro Nacional de Trasplantes; 2018.

5. Aburto S. Results 2015-2016, balance to two years of management in CENATRA. Bol Estad Inform. 2016;1:12-14.

6. Díaz-Chávez E, Orozco-Mosqueda A, Soel-Encalada J, Alemán-Suárez, D, Pérez-Granados, Gontes-Godínez J, et al. Initial report on the Results of the Kidney Transplant Program at the Bajio Regional High Specialty Hospital. Transplant Proc. 2016;48:620-624.

7. Marino-Vázquez LA, Sánchez-Ugarte R, Morales-Buenrostro LE. Kidney transplantation: consecutive one thousand transplants at National Institute of Medical Sciences and Nutrition Salvador Zubirán in México City. Rev Invest Clin. 2011;63:6-13.

8. Martínez-Mier G, Ávila-Pardo S, Irigoyen-Castillo A, Rodríguez-Fernán$\operatorname{dez} A$, Jiménez-López L, Varela-Pérez V. Analysis of the survival to 10 years of kidney transplant in the Hospital of High Specialty of Veracruz. Rev Mex Trasplant. 2016;5:113-119.

9. Mancilla-Urrea E, Aburto-Morales S, Kasep-Bahena J, Rodríguez-Castellanos F. Kidney transplant program at the Instituto Nacional de Cardiología Ignacio Chávez. Rev Invest Clin. 2011;63:14-18.

10. Reyes-Acevedo R, Romo-Franco L, Delgadillo-Castañeda R, Orozco-Lozano I, Melchor-Romo M, Gil-Guzmán E, et al. Renal transplantation program at the Centenario Hospital Miguel Hidalgo in Aguascalientes, México. Rev Invest Clin 2011;63:30-37.

11. González-Audiffred N, Alfaro-Abúndiz F, Aranda-Verástegui F, Isordia-Segovia J, Chevaille-Ramos A, Montes-De Oca-Arce J, et al. Experience in kidney transplantation from 1999 to 2011 at the Hospital Central Dr. Ignacio Morones Prieto, San Luis Potosi. Rev Invest Clin. 2011;63:38-43.

12. Mondragón-Ramírez G, Bochicchio-Riccardelli T, Bernal-Flores L, León-Rojas G, Martínez-Hernández J, Orozco-Tapia L, et al. Kidney transplant program: Mexican Institute of Transplants. Model of synergy between private hospital and private assistance foundations. Rev Invest Clin. 2011;63:44-49.

13. Martín-Escobar E. The Spanish Renal Registry: 2013 report and evolution from 2007 to 2013. Nefrologia. 2016;36:97-120.

14. Guirado L, Vela-Vallespín E, Clèries M, Díaz J, Facundo C, García-Maseta R. Why living-donor renal transplant yields better outcomes than cadaver renal transplant? Nefrologia. 2008;28:159-167.

15. Valdivia J, Gutiérrez C, Delgado E, Daymiris F, Treto J, Fernández I. Survival in kidney transplant with living donor transplant and cadaver donor. Investigaciones Medicoquirúrgicas. 2011;3:102-110.

16. Castañeda D, López L, Martín I, Martín R, Lozano E. Living kidney donors and kidney transplantation: a general overview. Urol Colomb. 2014;23:205-213. 